\title{
CARACTERIZAÇÃO DOS CLIENTES EM DIÁLISE PERITONEAL AMBULATORIAL CONTÍNUA-CAPD DA REGIÃO SUL DO BRASIL
}

Juliana Graciela Vestena Zillmer¹, Eda Schwartz², Vanda Maria da Rosa Jardim³ ${ }^{3}$, Rosani Manfrin Muniz' ${ }^{4}$, Maria Emilia Nunes Bueno ${ }^{5}$, Aline Machado Feijó ${ }^{6}$

RESUMO: Objetivo: descrever o perfil dos clientes em tratamento por Diálise Peritoneal Ambulatorial Contínua em um Serviço de Nefrologia da Região Sul do Brasil. Método: caráter epidemiológico, retrospectivo e descritivo; com a utilização do software Epi Info (6.04) para construção do banco de dados e análise. Resultados: população predominantemente feminina de cor branca, 50\% idosos, idade variando de 19 a 92 anos, $84,4 \%$ possuem baixa escolaridade, $46,9 \%$ são casados e 78\% dos clientes moram com familiar. Quanto à atividade laboral, 46,9\% não trabalham e 51,3\% recebem até 3 salários mínimos. Em relação aos fatores de risco, 31,3\% são fumantes e 28,1\% ingerem bebida alcoólica, 90,6\% têm hipertensão arterial sistêmica e 28,1\% diabete mellitus. O tratamento inicial foi a hemodiálise em $58 \%$ dos clientes e o tempo de permanência no programa é superior a dois anos. Conclusão: os resultados permitem caracterizar uma população em CAPD, possibilitando melhor planejamento do cuidado de enfermagem.

PALAVRAS-CHAVE: Insuficiência renal; Estudos epidemiológicos; Enfermagem; Diálise peritoneal.

\section{CHARACTERIZATION OF CLIENTS IN CONTINUOUS AMBULATORY PERITONEAL DIALYSIS (CAPD) FROM SOUTH REGION OF BRAZIL}

ABSTRACT: Objective: to describe the profile of clients in treatment for CAPD in a Department of Nephrology in the South region of Brazil. Method: epidemiological, retrospective and descriptive character, with the use of the software Epi Info (6.04) for both construction of the database and analysis. Results: predominantly white female population, $50 \%$ elderly, $84.4 \%$ have low education, $46.9 \%$ are married and $78 \%$ of the clients live with their family. Regarding to the laboral activity, 46,9\% don't work and 51,3\% receive up to 3 minimum wages. Regarding to the risk factors, 31,3\% smoke and $28,1 \%$ use alcohol, $90,6 \%$ have systemic arterial hypertension and $28,1 \%$ have diabetes mellitus. The initial treatment was the hemodialisis in $58 \%$ of the clients, and the time of permanence in the program is superior to two years. Conclusion: the results allow characterizing a population in CAPD, making possible better planning of nursing care.

KEYWORDS: Renal insufficiency; Epidemiologic studies; Nursing; Peritoneal dialysis.

\section{CARACTERIZACIÓN DE LOS CLIENTES EN DIÁLISIS PERITONEALAMBULATORIAL CONTINUA (CAPD) DE LA REGIÓN SUR DEL BRASIL}

RESUMEN: Objetivo: describir el perfil de los clientes en tratamiento por la CAPD en un Servicio de Nefrología de la región Sur del Brasil. Método: carácter epidemiológico, retrospectivo y descriptivo, con el uso del software Epi Info (6.04) para la construcción de base de datos y análisis. Resultados: predominio de población femenina de piel clara, 50\% ancianos, 84,4\% tienem bajo nivel de educación, 46,9\% son casados y 78\% de los clientes viven con la familia. Cuanto a los puestos de trabajo, 46,9\% no trabajan y 51,3\% reciben hasta 3 salarios mínimos. Cuanto a los factores de riesgo, 31,3\% son fumadores, 28,1\% ingieren bebida alcohólica, 90,6\% tienem hipertensión y 28,1\% tienem diabetes mellitus. El tratamiento inicial fue hemodiálisis en 58\% de los clientes, y lo tiempo de permanencia en el programa es superior a dos daños. Conclusión: los resultados permiten caracterizar una población de CAPD y posibilitan una mejor planificación de los cuidados de enfermería.

PALABRAS CLAVE: Insuficiencia renal; Estudios epidemiologicos; Enfermería; Diálisis peritoneal.

\footnotetext{
${ }^{1}$ Enfermeira. Mestranda em Enfermagem da Faculdade de Enfermagem e Obstetrícia da Universidade Federal de Pelotas-UFPel. Integrante do Núcleo de Condições Crônicas e suas Interfaces-Nuccrin.

${ }^{2}$ Doutora em Enfermagem. Professora da Faculdade de Enfermagem e Obstetrícia da UFPel. Líder do Nuccrin.

${ }^{3}$ Doutora em Enfermagem. Professora da Faculdade de Enfermagem e Obstetrícia da UFPel.

${ }^{4}$.Doutora em Enfermagem. Professora da Faculdade de Enfermagem e Obstetrícia da UFPel. Integrante do Nuccrin.

${ }^{5}$ Acadêmica de Enfermagem da Faculdade de Enfermagem e Obstetrícia da UFPel.

${ }^{6}$ Acadêmica de Enfermagem da Faculdade de Enfermagem e Obstetrícia da UFPel.
}

Autor correspondente:

Juliana Graciela Vestena Zillmer

Avenida Duque de Caxias, 170 - 96030-000 - Pelotas-RS

Recebido: 10/12/08

E-mail:julilmer@gmail.com

Aprovado: 26/06/09

Cogitare Enferm 2009 Abr/Jun; 14(2):318-23 


\section{INTRODUÇÃO}

A Doença Renal Crônica-DRC é uma enfermidade de elevada taxa de mortalidade e morbidade no Brasil e no mundo, implicando em altos custos sociais e econômicos ${ }^{(1)}$. O número de indivíduos com a doença aumenta de forma significativa, sendo considerado um grave problema de saúde pública, o que levou alguns pesquisadores a descreverem como a nova epidemia do século $\mathrm{XXI}^{(2-3)}$.

Em 2006 mais de 70.000 pacientes dependiam da terapia renal substitutiva, seja diálise (hemodiálise ou diálise peritoneal) ou transplante renal, com gasto anual aproximado de dois bilhões de reais. A previsão é de que o número de pacientes que venham a necessitar de alguma modalidade de substituição duplique nos próximos cinco anos, ultrapassando 125 mil casos em 2010, devido ao crescente número de grupos de risco com hipertensão arterial sistêmica e diabete mellitus ${ }^{(1)}$.

A doença renal crônica consiste em perda progressiva e, geralmente, irreversível da função dos rins, glomerular, tubular e endócrina. Em sua fase mais avançada, chamada de fase terminal ou estágio 5, os rins não conseguem mais manter a homeostasia do meio interno do organismo, necessitando de terapia renal substitutiva, sendo um deles a diálise peritoneal ambulatorial contínua-CAPD ${ }^{(4-5)}$.

A CAPD se caracteriza pela infusão de uma solução salina com dextrose na cavidade peritoneal por meio de um cateter implantado intra-abdominal. A solução entrará em contato com o peritônio, e por ele será feita a retirada das substâncias tóxicas do sangue $^{(6)}$. Nesta modalidade, o cliente é o principal responsável pela qualidade de seu tratamento, ou seja, realizar o autocuidado. A educação deste e inclusive de sua família para adoção de hábitos de higiene adequados e a correta realização do procedimento (troca das bolsas) constituem importantes medidas na prevenção de complicações como as peritonites. Dessa forma, o profissional de saúde, mais especificamente o enfermeiro, desempenha importante papel em CAPD, na medida em que ele deve contribuir para o estabelecimento de uma técnica segura e confiável, reduzir o número de internações e complicações, estimular e reforçar o processo de aprendizagem e conseguir o maior grau de reabilitação do cliente ${ }^{(7)}$.

Torna-se imprescindível conhecer o perfil dos clientes em tratamento dialítico, para que os profissionais possam planejar ações para o indivíduo no sentido de promover saúde, diminuir os riscos de complicações provenientes do tratamento por CAPD, e consequentemente proporcionar melhor qualidade de vida aos clientes dependentes desta terapia.Desta forma, o estudo teve como objetivo descrever o perfil dos clientes em tratamento por CAPD.

\section{MÉTODOS}

Trata-se de um estudo de caráter epidemiológico, descritivo e retrospectivo, envolvendo clientes cadastrados no programa de CAPD em um Serviço de Nefrologia de um município de porte médio da Região Sul do Brasil. O referido Serviço tem em seu espaço a presença de acadêmicos de enfermagem de uma Universidade Federal, devido a um Projeto de Extensão realizado desde o ano de 1992. Por meio deste projeto são desenvolvidas atividades administrativas, assistenciais, educacionais e de pesquisa centradas no doente renal crônico e sua família.

A população constituiu-se de indivíduos com doença renal crônica de ambos os sexos, de diferentes classes sociais e raças, submetidos à diálise peritoneal ambulatorial contínua, no período de agosto de 2006 a julho de 2007.

Neste período o Serviço de Nefrologia teve um total de 73 clientes submetidos a esta modalidade. No momento da realização da coleta, 46 clientes estavam em tratamento por CAPD e 27 já haviam saído do programa devido transferência para a modalidade de hemodiálise-HD, alta e outros foram a óbito.

Dos 46 clientes em tratamento e acompanhamento no Serviço, apenas 39 foram entrevistados, os outros sete não participaram da entrevista, pois quatro clientes apresentavam instabilidade clínica, dificuldade de compreensão e comunicação, dois não aceitaram participar e um cliente estava viajando para outro Estado.

Em relação aos dados dos 27 clientes que representam a saída do programa (óbito, transferências para HD e alta) os dados de 25 foram obtidos em prontuários, sendo que dois não foram disponibilizados por problemas administrativos.

Portanto, fizeram parte do estudo 64 clientes. Destes, 39 foram entrevistados e dados de 25 clientes foram obtidos em prontuários. Os clientes que cumpriam os critérios de inclusão para entrevista foram convidados a participar da pesquisa e, mediante aceitação, foi solicitado que assinassem o Termo de Consentimento Livre e Esclarecido.

Os questionários aplicados foram codificados 
pelo pesquisador. Construiu-se um banco de dados no software Epi Info (versão 6.4), no qual os questionários sofreram dupla digitação e limpeza das informações, sendo que estas também foram analisadas por este software. A análise univariada buscou descrever o perfil dos clientes em tratamento por CAPD no Serviço em estudo, utilizando-se de proporções e médias conforme o tipo de variável.

O projeto de pesquisa foi aprovado pelo Comitê de Ética e Pesquisa do Hospital Sociedade Portuguesa de Beneficência de Pelotas.

\section{RESULTADOS}

Foi realizada a caracterização sóciodemográfica dos 64 clientes, conforme Tabelas 1 e 2 .

Segundo os dados apresentados na Tabela 1, no que se refere a variável sexo, a população predominante é feminina (67,2\%). Na variável idade dos clientes 50\% foi de 60 a 92 anos, portanto população idosa, mas 50\% dos clientes estão na faixa etária dos 19 a 59 anos compondo o grupo dos economicamente ativos, semelhante ao encontrado em outro estudo ${ }^{(8)}$.

O estudo apresenta um dado social importante, pois a doença crônica atinge geralmente uma população em idade economicamente ativa, gerando gastos nas áreas sociais devido às aposentadorias precoces, gastos ambulatoriais e medicamentosos ${ }^{(9)}$. Quanto à cor, predominou a branca $(73,4 \%)$, também evidenciada em estudo realizado em um Serviço de Nefrologia no Estado de São Paulo com 217 clientes, cujo objetivo era caracterizar os pacientes com insuficiência renal crônica em tratamento dialítico ${ }^{(10)}$. Quanto à procedência, predominam os residentes do meio urbano (65,6\%), sendo todos pertencentes a municípios da Região Sul do Rio Grande do Sul.

Um dos fatores considerados importantes para selecionar um paciente para o programa de CAPD é a vontade referida pelo cliente de assumir seu autocuidado. Entretanto, o grau de instrução também é relevante, pois possibilita que o cliente receba informações por escrito auxiliando na compreensão das orientações no que se refere ao processo de comunicação e educação em saúde ${ }^{(11)}$.

O estudo constatou que $84,4 \%$ dos clientes possuem algum grau de escolaridade, o que possibilita ao enfermeiro ampliar o treinamento para a realização do tratamento por CAPD, fornecendo ao cliente materiais instrucionais como, por exemplo, folders, vídeos, além de orientações verbais abordando a prevenção de complicações referentes à modalidade terapêutica e a promoção de hábitos saudáveis.

Tabela 1 - Distribuição dos clientes em tratamento por diálise peritoneal ambulatorial contínua, segundo características sócio-demográficas na Região Sul do Brasil, 2007

\begin{tabular}{|c|c|c|}
\hline Características sócio-demográficas & n. & $\%$ \\
\hline \multicolumn{3}{|l|}{ Sexo } \\
\hline Masculino & 21 & 32,8 \\
\hline Feminino & 43 & 67,2 \\
\hline \multicolumn{3}{|l|}{ Idade } \\
\hline 19 - 39 anos & 8 & 12,5 \\
\hline $40-59$ anos & 24 & 37,5 \\
\hline $60-92$ anos & 32 & 50,0 \\
\hline \multicolumn{3}{|l|}{ Cor da pele } \\
\hline Branca & 47 & 73,4 \\
\hline Negra & 12 & 18,8 \\
\hline Parda & 5 & 7,8 \\
\hline \multicolumn{3}{|l|}{ Escolaridade } \\
\hline Analfabeto & 8 & 12,5 \\
\hline Ensino fundamental & 40 & 62,5 \\
\hline Ensino médio & 10 & 15,6 \\
\hline Ensino superior & 4 & 6,3 \\
\hline Não informado & 2 & 3,1 \\
\hline \multicolumn{3}{|l|}{ Situação conjugal } \\
\hline Casado & 30 & 46,9 \\
\hline Solteiro & 7 & 10,9 \\
\hline Viúvo & 22 & 34,4 \\
\hline Separado & 2 & 3,1 \\
\hline Relação estável & 2 & 3,1 \\
\hline \multicolumn{3}{|l|}{ Com quem mora } \\
\hline Sozinho & 4 & 6,3 \\
\hline Familiar & 50 & 78,1 \\
\hline Não informado & 10 & 15,6 \\
\hline
\end{tabular}

Quanto à situação conjugal, predominaram os clientes casados (46,9\%), seguido de viúvos (34,4\%). Observando os dados na Tabela 1, majoritariamente $(78,1 \%)$ residem com o familiar o que reforça o papel fundamental da família como cuidador, desde o diagnóstico da doença renal e ao tratamento. À medida que ocorre a evolução da doença, o paciente passa a apresentar dificuldades físicas que o impedem de realizar 
de forma autônoma seus compromissos, inclusive aqueles relacionados à terapia e ao autocuidado; o que exige o comprometimento e dedicação da família ${ }^{(12)}$.

A situação sócio-econômica do cliente e família é um fator a ser considerado, pois dele dependerá, na maioria das vezes, a continuidade do tratamento ${ }^{(12)}$. Em relação à atividade laboral, 46,9\% dos clientes não trabalham. O mesmo foi evidenciado em um estudo constatando que, de 43 clientes em tratamento dialítico, 54,55\% não trabalhavam por não apresentar condições físicas para realizar atividade laboral ${ }^{(13)}$. Em se tratando da renda familiar, 51,6\% dos clientes recebe de 1 a 3 salários mínimos regionais (o salário mínimo regional é de $\mathrm{R} \$ 477,90)$. A média de pessoas que dependem desta renda é de 3 indivíduos. Assim, os achados demonstram o baixo poder aquisitivo da população estudada. Tal fato pode vir a influenciar o acesso a medicamentos e materiais não fornecidos pelo Sistema Único de Saúde-SUS, o que favorece o aparecimento de complicações que aceleram a progressão da doença renal crônica.

Os dados encontrados indicam que esta é uma população mais vulnerável, podendo-se inferir que, frente ao padrão de escolaridade, a possibilidade de acesso aos postos de trabalho fica restrito a atividades de grande impacto físico. Soma-se a isso uma característica comum encontrada em estudos epidemiológicos em saúde do trabalhador que é o viés do trabalhador sadio, ou seja, para alguém se tornar trabalhador não deve ter doenças ${ }^{(14)}$.

Tabela 2 - Distribuição dos clientes em tratamento por diálise peritoneal ambulatorial contínua, segundo características socioeconômicas na Região Sul do Brasil, 2007

\begin{tabular}{lcc}
\hline Características sócio-econômicas & n. & $\%$ \\
\hline Trabalho & & \\
Sim & 9 & 14,1 \\
Não & 30 & 46,9 \\
Não informado & 25 & 39,1 \\
Renda & & \\
1 - 3 salários & 33 & 51,6 \\
+ de 3 salários & 7 & 11 \\
Não informado & 24 & 37,5 \\
\hline
\end{tabular}

Tabela 3 - Distribuição dos fatores de risco para doença renal crônica e doenças de base dos clientes em tratamento por diálise peritoneal ambulatorial contínua na Região Sul do Brasil, 2007

\begin{tabular}{lcc}
\hline Caracterização dos fatores de risco & n. & $\%$ \\
\hline Tabagismo & 20 & 31,3 \\
Sim & 19 & 29,7 \\
Não & 25 & 39 \\
Não informado & & \\
Álcool & 18 & 28,1 \\
Sim & 20 & 31,3 \\
Não & 26 & 40,6 \\
Não informado & 58 & 90,6 \\
Hipertensão arterial sistêmica & 18 & 28,1 \\
Diabetes mellitus & 13 & 20,3 \\
Cardiopatias & 7 & 10,9 \\
Litíase renal & 5 & 7,8 \\
Lupus erimatoso sistêmico & \\
\hline
\end{tabular}

Em relação à utilização do tabaco, verificou-se que $31,3 \%$ dos clientes são fumantes e $28 \%$ ingerem algum tipo de bebida alcoólica. Dados semelhantes foram encontrados em um estudo com 102 clientes, verificando-se que 36,3\%, em início de diálise, tinham o hábito de fumar ${ }^{(8)}$. Estudo epidemiológico realizado sugere que o tabagismo piora a sobrevida de clientes em tratamento dialítico, diminui $72 \%$ em dois anos e $40 \%$ em seis anos nos fumantes; quando acompanhado de hipertensão arterial, estes dados são ainda mais alarmantes $^{(15)}$.

A hipertensão arterial sistêmica-HAS representou a principal causa associada à doença renal crônica, estando presente em mais da metade (90,6\%) dos clientes estudados. A prevalência de HAS nesta população apresentou-se relativamente alta quando comparada a outros estudos ${ }^{(1,8,10)}$.

A HAS constitui-se em um dos maiores problemas de saúde pública do Brasil e de outros países, encontrando-se entre as morbidades mais frequentes do adulto e apresentando-se como uma das principais causas para a doença renal crônica. A sua detecção e tratamento devem ser prioridade para reduzir a morbimortalidade ${ }^{(4,9)}$.

De acordo com os dados, 28\% dos clientes apresentavam diabetes mellitus, semelhante ao encontrado no estudo ${ }^{(16)}$. A prevalência de diabete no Brasil varia de $18 \%$ na Região Norte até $27 \%$ na Região Sul, sendo a segunda causa de $\mathrm{DRC}^{(17)}$. Ressalta-se que a presença de diabete aumenta o risco de infecções, podendo estar associada à incidência de peritonite, que é uma das principais complicações do 
tratamento por CAPD ${ }^{(11)}$.

Foram identificadas outras doenças de base, representando $41 \%$, tais como: depressão, artrite reumatóide, doença severa óssea; dislipidemia; doenças infecto-contagiosas como hepatite B e tuberculose.

No que se refere à terapia de substituição renal, a Tabela 4 apresenta a distribuição dos clientes conforme a modalidade de tratamento inicial e o tempo de permanência em CAPD.

Tabela 4 - Distribuição dos clientes segundo tratamento inicial e tempo de permanência no programa de diálise peritoneal contínua e tratamento inicial, na Região Sul do Brasil, 2007

\begin{tabular}{lcc}
\hline Características do tratamento & n. & $\%$ \\
\hline Tratamento inicial & & \\
Hemodiálise & 37 & 58 \\
CAPD & 27 & 42 \\
Tempo para CAPD & & \\
Menos de 12 meses & 19 & 29,7 \\
1 a 2 anos & 14 & 22 \\
2 a 5 anos & 20 & 31,3 \\
+ de 5 anos & 11 & 17 \\
\hline
\end{tabular}

Verificou-se que $58 \%$ dos clientes tiveram como tratamento de primeira escolha a hemodiálise. Desta forma, com os achados concluiu-se que a maioria dos clientes tem como primeira opção a hemodiálise e demora a ingressar no CAPD, e só a praticam quando há necessidade de outra modalidade de tratamento. Isto pode ocorrer devido, na maioria das vezes, à falência de acesso vascular ou intolerância ao método hemodialítico, ou seja, iniciaram a diálise peritoneal somente quando tiveram problemas ${ }^{(16)}$.

Quanto ao tempo de permanência no programa de CAPD, observou-se que $31,3 \%$ dos clientes permaneceram por um período de dois a cinco anos, seguido de $29,7 \%$ a menos de um ano de permanência no programa. Estudo realizado comparando o tempo do diagnóstico da doença com o tempo de tratamento com o CAPD, constatou que $57,1 \%$ realizaram esta terapia há mais de 5 anos e $47,6 \%$ dos clientes praticam a modalidade CAPD há mais de um ano ${ }^{(16)}$.

\section{CONCLUSÕES}

Apesar de este estudo apresentar limitações como, por exemplo, a utilização de prontuários para a coleta de alguns dados, em que por vezes os registros não estão completos, e o pequeno número de clientes estudados, acredita-se que o presente estudo atingiu seu objetivo e ampliou o conhecimento quanto ao perfil dos clientes cadastrados no programa de CAPD de um Serviço de Nefrologia da Região Sul do Brasil, permitindo um melhor planejamento frente às necessidades reais dos clientes.

A partir dos dados apresentados, constatou-se que o perfil da população em tratamento substitutivo por CAPD é predominantemente feminina, de cor branca, idosa e, ainda, possuem baixa escolaridade. Com relação à atividade laboral, a maioria não trabalha e tem renda de até três salários mínimos regionais. Como fatores de risco, o tabagismo e o alcoolismo estão presente com percentual semelhante. A hipertensão arterial sistêmica e o diabetes mellitus são constados como sendo os principais fatores de risco para a doença renal crônica. Com isto, reforça-se a importância dos profissionais na Atenção Básica de Saúde, no monitoramento e acompanhamento de grupos de risco, como hipertensos e diabéticos.

Para o planejamento da assistência em saúde e realização do cuidado de enfermagem é necessário conhecer as características da população assistida, para se obter um diagnóstico da realidade local e contribuir na redução das complicações da doença renal crônica e do tratamento por CAPD.

\section{REFERÊNCIAS}

1. Sesso R. Epidemiologia da doença renal crônica no Brasil. In: Barros E, Manfro RC, Thomé FS, Gonçalves LFS. Nefrologia: rotinas, diagnóstico e tratamento. Porto Alegre: Artmed; 2006. p. 39-46.

2. Bastos MG, Carmo WB, Abrtita RR, Almeida EC, Mafra D, Costa DMN et al. Doença renal crônica: problemas e soluções. J Bras Nefrol. 2004;26(4):202-15.

3. Kirsztajn GM. Previna-se: uma idéia que está dando certo. J Bras Nefrol. 2007;1(Suppl 1):2-8.

4. Romão Júnior JE. Doença renal crônica: definição, epidemiologia e classificação. J Bras Nefrol. 2004;26 (Suppl 1):1-3.

5. Thomé FS, Gonçalves LFS, Manfro RC, Barros E. Doença Renal Crônica. In: Barros E, Manfro RC, Thomé FS, Gonçalves LFS. Nefrologia: rotinas, diagnóstico e tratamento. Porto Alegre: Artmed; 2006. p. 381-404.

6. Fermi MRV. Manual de diálise para enfermagem. São Paulo: MEDSI; 2006. 
7. Figueiredo AEPLF. Enfermagem e diálise peritoneal. In: Barros E, Manfro RC, Thomé FS, Gonçalves LFS. Nefrologia: rotinas, diagnóstico e tratamento. Porto Alegre: Artmed; 2006. p. 545-56.

8. Barbosa DA, Gunji CK, Bittencourt AGSB, Diccini FV, Vianna L AC. Co-morbidade e mortalidade de pacientes em início de diálise. Acta Paul Enferm. 2006;19(3):304-9.

9. Lessa I. Doenças crônicas degenerativas. In: Rouquayrol MZ. Epidemiologia e saúde. $5^{\mathrm{a}}$ ed. Rio de Janeiro: Medsi; 1999. p. 411-5.

10. Ribeiro RCHM, Oliveira GASA, Ribeiro DF, Bertolin DC, Cesarino LCEQL, Oliveira SM. Caracterização e etiologia da insuficiência renal crônica em unidade de nefrologia do interior do Estado de São Paulo. Acta Paul Enferm. 2008;21(n. esp):207-11.

11. Jacobowski JAD, Borella R, Lautert L. Pacientes com insuficiência renal crônica: causas de saída do programa de diálise peritoneal. Rev Gaúcha Enferm. 2005;26(3):38191 .

12. Carreira L, Marcon SS. Cotidiano e trabalho: concepções de indivíduos portadores de insuficiência renal crônica e seus familiares. Rev Latino-Am Enferm. 2003 Nov/Dez;11(6):823-31.

13. Lara EA, Sarquis LMM. O paciente crônico e sua relação com o trabalho. Cogitare Enferm. 2004;9(2):99-105.

14. Brandão AG, Horta BL, Tomasi E. Sintomas de distúrbios osteomusculares em bancários de Pelotas e região: prevalência e fatores associados. Rev Bras Epidemiol. 2005;8(3):295-305.

15. Biernat JC. Sobrevida em hemodiálise. In: Cruz J, Barros RT, Sesso RCC, David Neto E, Suassuna JHR, Heilberg IP, et al, coordenadores. Atualidades em nefrologia 3. São Paulo: Savier; 1994. p. 155-159.

16. Silva HG, Silva MJ. Motivações do paciente renal para a escolha a diálise peritoneal ambulatorial contínua [periódico na Internet]. Rev Eletron Enferm. 2003 [acesso em 2008 Nov 20] 5(1):10-14. Disponível: http:// www.revistas.ufg.br/index.php/fen.

17. Sociedade Brasileira de Nefrologia. Censo 2006 para o tratamento dialítico crônico. [acesso em 2008 Jul 30] Disponível: http://www.sbn.org.br/Censo/2006/ censoSBN2006.ppt. 